\title{
The Best of Possible Worlds: A Testable Claim of Choice
}

\section{WILLIAM C. LANE}

\begin{abstract}
Leibniz said that the universe, if God-created, would exist at a unique, conjoint, physical maximum: Of all possible worlds, it would be richest in phenomena, but its richness would arise from the simplest physical laws and initial conditions. Using concepts of "variety" and algorithmic informational complexity, Leibniz' claim can be reframed as a testable theory. This theory predicts that the laws and conditions of the actual universe should be simpler, and the universe richer in phenomena, than the presence of observers would require. Tegmark has shown that inhabitants of an infinite multiverse would likely observe simple laws and conditions, but also phenomenal richness just great enough to explain their existence. Empirical observations fit the claim of divine choice better than the claim of an infinite multiverse. The future of the universe, including its future information-processing capacity, is predicted to be endless.
\end{abstract}

Key words: Leibniz; Fine tuning; Anthropic principle; Multiverse; Emergent complexity; Observer effects; Heat death; Algorithmic informational complexity; Design; Copernican principle

If only we could sufficiently understand the order of the universe, we should find that it surpasses all the desires of the wisest, and that it is impossible to make it any better than it is. Gottfried Wilhelm Leibniz ${ }^{1}$

The a priori probability that a randomly generated universe would be capable of supporting complex life is, by all accounts, vanishingly small. ${ }^{2}$ Yet the values of many physical constants seem tuned to give our actual Universe just that capability. ${ }^{3}$ Scientists, philosophers, and theologians have advanced many possible explanations for this "fine-tuning coincidence." ${ }^{4}$ While these explanations vary widely, they fall into two broad categories. We can label those categories "choice" and "multiverse."

In the choice category are theories that say some physical process, selection principle, or creator deity picked our physical laws and initial conditions out of the infinite possibilities that might have come to exist. Our special set of laws and conditions therefore exists for a reason. Perhaps it was the only set chosen, or perhaps it is one of a limited number of chosen sets. Not all "choice" theories involve a "Chooser." While some are plainly theistic, others appeal to the Darwinian notion that choice can result from mindless, natural causes. ${ }^{5}$ However, 
all choice theories agree on this: For some reason only one or a (relative) handful of sets of laws and conditions actually exist. Vastly many other possible sets of laws and conditions, in fact an infinite range of possibilities, might have existed a priori, but for some reason do not.

The competing multiverse theories suggest that our "visible universe"everything that we can see-is only a tiny, atypical region in an infinitely vast "Multiverse" (set of universes). A limitless number of other regions actually exist elsewhere in the Universe. Wherever these regions may be, and however they may have arisen, they exhibit an equally limitless range of differing, but actually existent physical laws and conditions. As a result, according to David Lewis, "There are so many other worlds... that absolutely every way that a world could possibly be is a way that some world is..."6 Almost none of these "worlds" support life or anything else of interest, but our rare region does. We find ourselves in this life-supporting region because (a) it must exist along with all the others, and (b) we must inhabit a region that supports our existence. ${ }^{7}$

Scientific opinion has until recently given grudging assent to the multiverse principle. ${ }^{8}$ However, a recent paper by Stoeger, Ellis, and Kirchner ("SEK") strongly suggests that some principle of choice must be at work in the Universe." SEK argues that:

1. There are strong mathematical and logical reasons to doubt that an actually realized, physical Universe could be infinite in any meaningful sense.

2. In any event, there is no unique, actually infinite Universe. One possible Universe, though infinite in extent, might contain many "Type A" regions but none of "Type B." Another equally infinite Universe might consist entirely of "Type B" regions. A Universe that is infinite in extent need not contain an infinite diversity of regions. It need not occupy all of possibility space.

3. Thus, even in an actually infinite Universe, something-some person, process, or principle-must distribute laws and conditions in its regions one way rather than another. We cannot dispense with choice.

These arguments suggest that choice-based approaches deserve more scrutiny than they have received. This paper will examine one such approach, advanced by Leibniz three centuries ago. This is his claim that a perfect God would only create the "best" of possible Worlds. ${ }^{10}$ As will be explained below, Leibniz suggested a sense of World merit under which it is possible to claim that there could be a best of possible Worlds. This paper will: (a) suggest that Leibniz' notion of World merit is subject to quantification and empirical test; and (b) advance considerations for believing that our actual World is the best of possible Worlds in this sense.

\section{Leibniz' richest/simplest claim}

Leibniz was probably the first to notice that the structure of the Universe is not logically required, that everything, including the laws of physics, might have been fundamentally different. ${ }^{11}$ Even "time, space and matter... might have received 
entirely other motions and shapes, and in another order."12 He wondered why, out of an infinite number of possibilities, the Creator chose to actualize this particular setup, the Universe that we know and inhabit. This question led him to a remarkable conjecture.

Leibniz envisioned God as possessing all perfections: infinite wisdom and power as well as perfect goodness. ${ }^{13}$ Because he believed that God would not act without a reason, he argued that God would actualize only the best of possible Worlds. "[T]his is the cause for the existence of the best: namely, that his wisdom makes it known to God, his goodness makes him choose it, and his power makes him produce it." 14

At least since Voltaire, Leibniz' critics have seen this claim as a valid argument to an "immensely implausible conclusion."15 Yet is the conclusion so implausible? Most critics never address the final term of Leibniz' argument, his specific description of the best of possible Worlds. Leibniz phrased that description in a number of ways: "[T]he most perfect World" is "the one which is at the same time the simplest in hypotheses and the richest in phenomena, as might be a line in geometry whose construction is easy and whose properties and effects are extremely remarkable and widespread."16 "It follows from the supreme perfection of God that in producing the Universe He chose the best possible plan, containing the greatest variety together with the greatest order...the greatest effect produced by the simplest means...."17 "[P]erfection is not to be located in matter alone, that is, in something filling time and space... rather, it is to be located in form or variety." ${ }^{\prime 18}$ The Leibnitian Universe (since the Universe is everything that God creates, there can only be one) would exhibit the greatest possible variety, richness and abundance of natural phenomena, but these would result from the simplest possible "hypotheses" (the Universe's physical laws and initial conditions).

Thus, Leibniz' metaphysical assumption of God's perfection led to a specific, physical description of the Universe. ${ }^{19}$ We can call that description Leibniz' richest/simplest claim. This "two-factor assessment of perfection" insists on both richness as an end and simplicity as a means. ${ }^{20}$ Each is equally important: "For the wisest mind so acts as far as is possible, that the means are also ends of a sort, i.e. are desirable not only on account of what they do, but on account of what they are."21 Leibnitian perfection is, "a matter of combining, harmonizing and balancing [these] two distinct factors" into a joint maximum. ${ }^{22}$

One intriguing point about this claim is its close agreement with descriptions of the actual Universe proffered by modern scientists. Freeman Dyson says that our simple laws seem designed to "make the universe as interesting as possible."23 Carl Sagan says our Universe is "lavish beyond imagining." ${ }^{24}$ Astronomer Mario Livio suggests a "cosmological aesthetic principle" involving simplicity, symmetry, and the Copernican principle. ${ }^{25}$ Murray Gell-Mann marvels at how, "[i]n an astonishing variety of contexts, apparently complex structures or behaviors emerge from systems characterized by very simple rules." ${ }^{26}$ Physicist A. Zee reports that the Universe exhibits, "unity and diversity, absolute perfection and boisterous dynamism, symmetry and lack of symmetry." ${ }^{27}$ Paul Davies says that 
the Universe seems to be governed by "very special laws that guarantee a trend toward greater richness, diversity and complexity through spontaneous selforganization...."28

\section{Quantifying simplicity and richness}

Another salient feature of Leibniz' richest/simplest claim is its potential to specify the single, best possible Universe from an infinite range of possibilities. No singlefactor criterion can do this. No possible Universe could contain "the most" phenomena; more could always be added. No possible Universe could be "happiest"; it could always be happier. By contrast, a Universe whose design requirement opposes two competing factors can (potentially) attain a maximum. In such a World, the need for laws and initial conditions to be simple prevents mere chaos from piling up, while the requirement that laws and conditions generate maximal richness prevents the World's "hypotheses" from being so simple as to be useless. Leibniz' claim thereby defeats the riposte that a search for the "best possible World" is like a search for the highest number. It suggest the possibility of a unique balance, a uniquely "best" World, ${ }^{29}$ or at least a uniquely best set of World-generating physical laws and initial conditions. ${ }^{30}$ However, can Leibniz' claim actually specify a quantitatively unique point of balance, or does it only make a qualitative suggestion? The answer turns on whether we can state it mathematically.

\section{Quantifying simplicity}

The standard measure of relative simplicity is "algorithmic information content" ("AIC"). The AIC of anything is just the length of the shortest set of computer instructions that, if followed, would completely generate, compute, or describe that thing. ${ }^{31}$ AIC can be measured in bits. The AIC of a wagon is the number of bits needed for a program that will print out a complete description of the wagon. The description of an automobile would require a longer program, one with a higher AIC. Calculations of AIC can yield different results depending on the hardware and software used and other extraneous factors, ${ }^{32}$ but these factors typically wash out when relative simplicity is at issue and the same approach is taken for all measurements. ${ }^{33}$

Our imperfect knowledge of physics and cosmogony poses a sharper limitation. To measure the AIC of our actual laws of physics and initial conditions, we first need to know what they are. Most physicists would say that we do not yet have the "final" laws of physics; nor can we precisely describe the Universe's first fractional second of existence. However, it should be possible to calculate the AIC of these "final" laws and conditions when (if) we someday discover them, and to compare that calculated quantity with the AIC of other possible sets of laws and initial conditions. AIC is therefore, in principle, a reasonable way to measure and compare the simplicity of our "hypotheses." 


\section{Quantifying richness}

Richness is a more complex subject. Indeed, complexity is an important aspect of richness. Today we say that a system is complex if it includes many parts that interconnect in intricate ways. A living cell is complex; a salt crystal is not. One characteristic of a complex system is its structured self-similarity. Small regions resemble bigger regions; discrete components and processes repeat, but rarely in the same way. A Universe of maximal complexity would be an elegant World, a blend of order and diversity. It would exhibit what Heisenberg called a "proper conformity of the parts to one another and to the whole." 34 A rich World in Leibniz' sense would be full of detailed, nonrepetitive, self-similar structure.

It would also be large. We can think of Leibnitian richness as "complexity plus," where the "plus" is size. A Leibnitian Universe would contain a vast number of complex phenomena, each interrelated in diverse ways to other phenomena, each a composite of many parts. All of these diverse, nested phenomena would owe their existence to simple and "sublime principles which show the wisdom of the Author in the order and perfection of his work." 35

Physicists Julian Barbour and Lee Smolin ("B\&S") have made a start on describing richness mathematically. ${ }^{36}$ They call their construct "variety." Here is how it works: Start with a single elementary particle. We can call the particles nearest to it, or with which it interacts most directly, its neighborhood. We can say that a system has higher variety if we need less information to distinguish each of its elementary constituents from all the other constituents of the system, by describing its neighborhood. Alternatively, a system has lower variety if we need more information to distinguish each constituent from the others by describing its neighborhood. In a low-variety system, each neighborhood has to be big to be unique. In a high-variety system, each neighborhood can be unique although it may be quite small. Variety diminishes if you have to go further afield to find a unique neighborhood; it increases with the total number of elementary constituents in the system as a whole. "The variety is a non-local and non-additive quantity, which can only be applied to a system as a whole. It measures, in a certain sense, how unique, one from another, the different parts of the system are." 37

B\&S show two ways to calculate the variety of a graph made of dots. The simpler way is to identify a subgraph with an arbitrary dot, $i$, as its starting point, then add to the subgraph each of $i$ 's immediately neighboring dots. Each added dot counts as a step. As the subgraph grows, map it against every other dot, $j$, in the original graph, where $j \neq i$. When you get a subgraph that does not map onto the neighborhood of a particular $j$, call the number of steps in that subgraph the relative indifference of $i$ and that $j$. When you get a subgraph that does not map against the neighborhood of any $j$, call the number of steps in that subgraph the absolute indifference $(R)$ of $i$, denoted as $R i$. The variety of the graph as a whole (treating every dot in succession as an $i$ ) then equals:

$$
\mathrm{V}=\sum_{i} 1 / R i
$$


To see how this might work, imagine that you need to identify every house in two different, equally sized cities. You cannot use street addresses or north-south directions; nor can you describe the houses, for they are all identical. You can, therefore, only describe a house in relation to its neighbors. To identify each house, you will need to describe its spatial relationship to every nearby house-how far apart they are, the angles between them and so on-and keep doing that in a widening circle until you have described a unique neighborhood, a set of relationships not replicated anywhere else in the city. Once you identify a unique neighborhood, you have specified the house at its center. You can then do the same thing for each other house in each city. Imagine that in one city the houses stand irregularly on narrow, winding streets. The other city has straight streets, rectangular intersections, and houses evenly spaced. Using the method described above, the first city would have higher variety. In the city with narrow, winding streets, you would have to look at fewer neighboring houses to find a unique neighborhood. Each group of houses in the high-variety city would tend to embody more information than an equal number of houses in the more regular city. When a system gets larger, its variety tends to increase ${ }^{38}$ In the example of a city, each additional house would add another " 1 " to the sum of the numerators in equation (1). In a large, high-variety system, tiny neighborhoods (e.g. molecules) might be identical, but would be embedded in larger, non-identical neighborhoods (e.g. rocks). Large, similar neighborhoods (e.g. human twins) would be non-identical by virtue of the smaller neighborhoods that compose them (e.g. their individual cells). B\&S conclude that variety "distinguishes highly structured, but asymmetric, configurations such as one finds in biological systems from both random configurations and [ordered] configurations such as crystals ...",39

\section{Objections to variety as a metric for richness}

We can use variety to measure the richness of a small, static system. Can we use it to measure the richness of the Universe? Two objections have been stated:

(a) The Universe is too complex to measure in this way. It has many different kinds of particles (electrons, photons, etc.), not just identical dots. Moreover, its elementary constituents are not really particles in Newton's "hard, massy" sense; they are more like events that are here one moment, then gone the next. Finally, the sheer number of particles and the diversity of their arrangements is incredibly vast. Given these complexities, we will never be able to map each particle's neighborhood against all the others, and therefore, cannot possibly calculate a number that describes universal variety.

(b) Variety does not consider the interactivity of a system's structures. For example, it does not consider the kinds of complex interactions that exist in biological systems. It ignores the sorts of emergent phenomena that make our Universe an interesting place. Because variety considers only the static arrangements of particles in a system, it might be higher in a well-stocked 
junkyard than in a similar area of forest. ${ }^{40}$ It therefore is not, even in principle, a useful measure of the richness of our Universe.

\section{Response to Objection A}

Objection A mistakes the role intended for variety. There is no suggestion that we measure the variety of the Universe with mathematical precision. The suggestion instead is that the calculability of variety in principle shows that Leibnitian "richness" has some definite, quantifiable meaning, such that the relative ability of possible sets of physical laws and initial conditions to generate maximal variety could be a criterion for selecting a particular set of laws and conditions for actualization. In principle, this criterion could partly explain why our laws and conditions are what they are.

This in itself is a large step forward. There have been only a few attempts to quantify richness and diversity, and those few have occurred in biology. For example, ecologists distinguish among alpha, beta, and gamma measures of diversity. ${ }^{41}$ Yet these measures count only the number of species. They do not account for diversity within a species, or for the diversity of galaxies, planets, or human invention. These ecological approaches are therefore not of much use in specifying a maximally rich universe. Variety in the B\&S sense is more useful precisely because of its "generic" quality. It does not depend on how we categorize things; it tries to get at the overall character of a system by examining the arrangements of its elementary particles. ${ }^{42}$

Moreover, while calculating the Universe's variety seems out of the question, estimating it does not seem impossible. We might analogize this problem to the task of determining the contents of a computer disk. Most of us could not decipher a disk by reading a printout of its 1's and 0's. Yet by employing a series of intermediate languages-assembler, then an operating language, and finally a higher-level application-we can make its contents emerge on our screen. In much the same way, further explorations of the concept of variety may show us how to determine the relative variety of large systems by observing their higher-order characteristics. ${ }^{43}$ Life, for example, may both provide the highest-variety systems in the Universe and act as the most prolific source of new, high-variety systems. If so, then the biofriendliest Universe would also be the highest in variety. As we learn about the role of the physical constants in the origin of life, the question of whether our Universe is the biofriendliest of all possibilities might someday seem to have an obvious answer. We could then decide whether our Universe is (or is not) the highest in variety of all possibilities without examining the configuration of its elementary particles.

Objection A's other concerns also seem surmountable. For example, we might focus on protons in estimating variety, regarding other particles as merely the means by which the system arranges protons. Alternatively, we could focus on fields as the Universe's fundamental elements; quantum physics handles the location and interrelation of fields with mathematical precision. The use of fields 
might require a different formalism than the one suggested by B\&S, but the prospect seems to pose no conceptual difficulties. ${ }^{44}$

While calculating the variety of the protons in a postage stamp would be a challenging task today, the point of focusing on variety is not to find a single, precise measure of the richness of our Universe. The objectives instead are (i) to assure ourselves that richness can have more than a merely subjective meaning, and (ii) to learn about the specific sorts of higher-level characteristics that we might expect in the maximally rich Universe. We can then compare our Universe with other possible Worlds with more confidence that we are focusing on fundamental rather than parochial characteristics.

\section{Response to Objection B}

Objection B is correct to a point. Variety as explained above describes the arrangement of particles in a static system. However, there are at least two different approaches to employing the notion of variety in a changing system. The first, proposed by $B \& S$, would be to regard the maximal variety configuration as, in some sense, the goal towards which the system tends to strive. We might then imagine that our laws of physics are the local results of a non-local potential energy function created by this global tendency toward high variety. ${ }^{45}$ A difficulty with this approach is that, while it captures certain features of the dynamical behavior of the Universe, it does not seem to capture others. $^{46}$

An alternative approach (suggested here) would preserve the ontological independence of our laws of physics, but would suggest that, along with our initial conditions, they mark the simplest path to maximal variety. This approach begins by restating equation 1 as follows:

$$
\mathrm{V}_{1}=\sum_{i} 1 / R i_{n}
$$

Here, $\boldsymbol{R}$ in this bold font represents the absolute indifference of $i$, not over the Universe at a single moment in time but over the whole history of the Universe captured at $n$ snapshots of time. The number of snapshots considered in this formula (represented by $n$ ) can be somewhat arbitrary, but must give a reasonable, overall depiction of the Universe over time, and must include a snapshot from each important stage of its history. The absolute indifference of $i$ in this equation would then reflect the smallest number of moves needed to distinguish the neighborhood of $i$, not only from all other neighborhoods, but also from $i$ 's own neighborhood at all later or earlier "snapshot" moments. Owing to this second requirement, a static system with high variety under equation (1) would display lower variety under equation (2) than a similar system that changes significantly over time.

A claim that our Universe has maximal variety over time could then be stated:

$$
\mathrm{V}_{1}>\mathrm{V}_{0}
$$


Here $V_{1}$ is the variety over time of our actual Universe and $V_{0}$ is the variety over time of any other, possible universe, calculated in the same way and using the same value for $n$. Unlike equation (1), expressions (2) and (3) capture the effects of system interactivity and emergent behaviors because only complex, dynamic processes could produce the continual changes that would be required to generate maximal variety over time. A junkyard will either remain a junkyard or else simply disintegrate. In either event, time will not significantly enhance its variety as measured in equation (2). In a forest, however, "endless forms most beautiful and most wonderful have been, and are being evolved. ${ }^{\prime 47}$ These evolving forms include not only new species, but also emergent differences among individuals and new patterns of cooperation and conflict. So long as biological processes continue to operate, the passage of time will increase the forest's variety over time under equation (2). This suggests that, over time, a universe containing life will have higher variety than a lifeless universe.

\section{Simplicity and richness}

We can now add Leibniz' conjoint requirement of maximal simplicity:

$$
\mathrm{V}_{1} / \mathrm{A}_{1}>\mathrm{V}_{0} / \mathrm{A}_{0}
$$

In this inequality, $V_{1}$ and $V_{0}$ are from expression (3), while $A_{1}$ represents the AIC of the laws of physics and initial conditions of our actual Universe and $A_{0}$ reflects the same quantity for any other possible universe. Expression (4) reflects Leibniz' insistence that our Universe must be better than any other possibility. "If there were not the best among all possible worlds, God would not have produced any." ${ }^{\prime 4}$ It also fits with Leibniz' insight that the "most perfect possible world is that which exhibits the greatest variety of its contents (richness of phenomena) consonant with the greatest simplicity of its laws." ${ }^{\prime 9}$ It balances and opposes simplicity and richness in an intuitively appealing way. ${ }^{50}$ Expression (4) shows that Leibniz' richest/simplest claim could pick out a single, uniquely "best" setup.

\section{Do we inhabit Leibniz' universe?}

The tautological fact that we must find ourselves in a region that supports our existence defeats many attempts to frame a persuasive argument from design out of the fine-tuning coincidence. The weak anthropic principle ("WAP") suggests that, regardless of where or how it originated, the Universe must be at least special enough to produce us. ${ }^{51}$ Since intelligent observers can (it seems) only exist under laws of physics similar to ours, it is, the argument runs, unremarkable that we find ourselves in a Universe governed by such laws. ${ }^{52}$

On the other hand, it would be remarkable if our Universe were governed by physical laws and initial conditions that met Leibniz' conjoint criterion of maximal 
simplicity and fecundity. Under any view of the Copernican principle, it seems unlikely that the human race or intelligent observers in general are so special that their existence requires maximal conditions. Instead, many different, relatively high-variety universes based on relatively simple "hypotheses" should be capable of supporting creatures like us. ${ }^{53}$ This intuition, if true, opens a conceptual gap between the Leibnitian Universe (governed by the richest/simplest principle) and the merely sufficient Universe predicted by the WAP. This gap offers a way to test Leibniz' claim that does not require either a perfect knowledge of the laws of physics or a detailed analysis of the arrangements of protons. If ours is the best of possible Worlds, then our laws of physics should be simpler than they need to be to produce us and our Universe should be richer (higher in apparent variety over time) than our presence would require. ${ }^{54}$

\section{Excess simplicity?}

Physicist Stephen Wolfram suggests that, "among all possible rules [of physics], the overwhelming majority will not be simple; they will instead tend to be almost infinitely complex." ${ }^{\prime 55}$ This is, Wolfram says, because any law of physics must take the form of some mathematical expression, and there are many more complex expressions than simple ones. If this logic is sound, and if we inhabit a random region in the multiverse (except that it lets us exist), then the laws of physics in our region should be quite complex.

This is not what we experience. In 1981, physicist E. J. Squires wrote a paper entitled, "Do we live in the simplest possible interesting world?"56 Squires assumed that the world would not be "interesting" without chemistry and stars, and that stars need nuclear fusion. Then he showed how the electromagnetic force, the weak and strong nuclear forces, and the properties of quarks, neutrinos, photons, and electrons might all derive from these two requirements in the simplest, most parsimonious way. Though Squires did not mention general relativity, the principle of relativity asserts that the laws of physics must be the same in all frames of reference. After discovering this principle, Einstein concluded that, "experience justifies our belief that nature is the realization of the simplest mathematical ideas that are reasonable.",57

Our initial conditions are even simpler. Davies describes the state of the Universe at the big bang as "a featureless ferment of quantum energy, a state of exceptionally high symmetry. Indeed, the initial state of the universe could well have been the simplest possible." ${ }^{\prime 58}$ The entire "featureless ferment" of our spacetime then expanded with extraordinary rapidity. As it expanded, quantum processes like those that continue to operate throughout the Universe formed tiny irregularities, which became the earliest ancestors of every later structure, from galaxies to philosophy textbooks.

This is a simple beginning, but the real story may be simpler still. Our Universe may have emerged out of absolutely nothing. Not just empty space, for "empty" space is not empty at all. Physicists call it a "false vacuum," densely packed with "dark energy," "the seat of various energetic processes." ${ }^{\prime 59}$ Inflationary big bang 
theory says the Universe emerged from a vastly emptier state, a true vacuum. Pagels explains:

The nothingness "before" the creation of the universe is the most complete void that we can imagine-no space, time, or matter existed... Yet this unthinkable void converts itself into a plenum of existence-a necessary consequence of physical laws... It would seem that even the void is subject to law, a logic that exists prior to time and space. ${ }^{60}$

No simpler conditions than these-"nothing" plus the laws of physics-are imaginable.

We could, it seems, exist in a universe where the laws of physics varied in certain inertial systems, or in a World where the weak nuclear force and electromagnetic force were not the same at the deepest level, or in a World that did not originate from "nothing." The fact that we must observe a universe that we can inhabit does not explain the striking simplicity of our "hypotheses."

Tegmark, however, has suggested a different explanation. ${ }^{61}$ Taking a bird's eye view of mathematics, he shows that it consists of a nested series of mathematical structures: Adding a metric to the structure called "topological spaces" generates the structure called "metric spaces"; combining tensor spaces with real manifolds creates the structure called "manifolds with tensor fields." ${ }^{62}$ In general, complex structures are just simple structures with added rules or requirements. Tegmark then makes two assumptions: (i) that physical existence is equivalent to mathematical existence (the infinite multiverse assumption); and (ii) that mathematical existence is merely freedom from contradiction. If these assumptions are true, and assuming that self-awareness is a function of complexity, then all categories of mathematical structure that are complex enough to contain "selfaware subsystems"-Tegmark calls them SAS's-must exist as physical realities for the SAS's (a/k/a "intelligent observers") they contain.

Tegmark goes on to note that, if his claims were true, most SAS's would find themselves in the mathematically simplest structures that could possibly produce them. Ironically, this is because simpler mathematical structures allow for more complexity. They impose fewer restrictions on possible combinations of their elements; they thereby allow more differing phenomena than structures with more rules. In Tegmark's view, an SAS is likely to find itself in a world governed by the simplest mathematics that can produce it, even though more complex mathematics might have produced it as well.

Leibniz made a closely related argument in support of his claim that God would choose the simplest possible "hypotheses." He said that simple rules let God "find room for as many things as it is possible to place together. If God had made use of other [more complex] laws, it would be as if one should construct a building of round stones, which leave more space unoccupied than ...they fill. ${ }^{\prime 63}$ Picture a fieldstone building of the kind common in Leibniz' day. The builders used various shapes and sizes of stones; in fact, they used whatever fit. Any individual stone would be likelier to find itself in a fieldstone building than in one for which stones are chosen according to a complicated 
rule or pattern. Leibniz claimed that our physical laws and initial conditions should be maximally simple so that the Universe could contain the greatest richness of phenomena. Tegmark claims that the "mathematical structure describing our world" should be "the most generic one that is consistent with our observations," i.e. with our ability to observe, precisely because most phenomena will be contained in the simplest regions capable of hosting them. ${ }^{64}$ Assuming that both these arguments are valid, the extreme simplicity of our physical laws cannot distinguish between the Leibnizian and Tegmarkian explanations. On the other hand, in every contemporary multiverse theory each constituent "pocket universe" begins from much the same initial conditions. With no diversity of origins from which to select, Tegmark's reasoning cannot explain the extreme simplicity of the big bang.

\section{Excess richness?}

Tegmark says that, if the multiverse exists, "Our observations [should be] the most generic ones that are consistent with our existence. ${ }^{\prime 65}$ If we live in a random, life-supporting region of the Tegmarkian multiverse, our world should be just rich enough to include us. ${ }^{66}$ Leibniz predicts just the opposite: We should find ourselves in the World containing the greatest possible richness of phenomena, of which we are not likely to be the supreme example. Thus, were we to discover richness greatly in excess of that needed for our existence, that discovery would strongly support Leibniz' claim.

Is there evidence of excess richness? Might we find more such evidence in the future? The remainder of this paper addresses these questions.

We might begin by considering the billions of galaxies that we can observe and the billions of stars they each contain. Hawking says that, "our solar system is certainly a prerequisite for our existence, as is an earlier generation of nearby stars in which heavy elements could have been formed by nuclear synthesis.... But there does not seem any necessity for other galaxies to exist, let alone the million million or so of them that we see..." ${ }^{\prime 67}$ The vast surfeit of galaxies looks like an example of excess structure. It seems that we could exist in a universe with a few hundred galaxies, or perhaps with just one.

The Universe is also larger than it needs to be. The geometry of the Universewhether space is flat or curved-does not determine its topology or the rate of its expansion. Because these are independently determined, the Universe could have been smaller than the region of space we observe.$^{68}$ If that had turned out to be the case, some galaxies we see in one direction from the Earth could be the same ones we see in the opposite direction. If the Universe were small enough, we might even see our own Milky Way galaxy as it once existed. This would not be a copy; it would be the real thing, our galactic home viewed down a vast, curving tunnel of light. Astronomers have found, however, that nature did not take this "small universe" path; the Universe appears to be larger than all of observable space. ${ }^{69}$ The extra richness this extra size requires-extra in the sense that it was not requisite to our existence-supports Leibniz' claim. 
More pointedly, we can think about the future. The WAP tells us nothing about the future. It says only that any universe we inhabit must have been capable of producing us, of getting us to where we are now. Leibniz' richest/simplest claim, by contrast, makes a definite prediction: The greatest possible richness is just the largest possible "quantity of reality." Since a longer-lived Universe means more change, and hence more "reality," the Universe should continue for as long as possible. This is a testable prediction, and the early results are in. For a long time astronomers thought the persistent inward tug of gravity might eventually overcome the force of the Hubble expansion. As the galaxies fell back into each other, their mutual gravities could result in a "big crunch," a moment when everything would smash into everything else, and that would be the end. We now know, however, that the Hubble expansion is accelerating. ${ }^{70}$ Our Universe seems set to grow forever in both size and duration. ${ }^{71}$

It has sometimes been suggested that this continuous expansion will end in a "heat death," a final state of maximum entropy (zero order) when nothing more can happen. ${ }^{72}$ However, this is not a necessary outcome. If the Universe expands at an optimal rate, it can continue experiencing new and interesting phenomena forever, never losing information.

This point is worth explaining. ${ }^{73}$ We can define order as the absence of disorder. Since there is more than one kind of order (living cells versus salt crystals), but only one kind of disorder (mathematical randomness/maximum entropy), defining order this way captures all kinds of order. Since information is a measure of order, we can define information as:

$$
\mathrm{I}=\mathrm{D}_{\max }-\mathrm{D}_{\text {actual }}
$$

According to equation (5), information (order) is present whenever the maximum possible disorder consistent with given constraints exceeds the actual amount of disorder. The size of the gap measures the amount of information (order) present.

Imagine a balloon full of compressed gas. The balloon is in the center of a large spherical room, which otherwise contains a vacuum. The gas in the balloon is in a state of maximum entropy. Gas molecules moving at different speeds and in different directions mix together randomly. No order is present until we burst the balloon. Then each molecule continues moving in the direction and at the speed it was already traveling, but with nothing to constrain it. Soon the molecules arrange themselves in a series of concentric spheres with the fastest molecules traveling most quickly towards the spherical wall of the room and slower ones lined up behind according to speed. In each concentric, speed-ranked sphere, each molecule lines up next to others traveling in nearly the same direction. Increasing the allowable disorder (letting the molecules spread out) has created very discernable order.

Now change this scenario: The spherical wall of the room starts out close to the balloon, but can expand smoothly away from it. Now the amount of order created will depend on the speed of the wall's expansion. If it expands faster than the gas molecules-keeping out of their way-the gas expansion will create order just as if the wall were not there. If the wall expands more slowly, the growing amount of actual disorder caused when molecules bump into it may keep up with the growing potential for disorder. If that happens, the system will generate no new order. 
The universal expansion, in tandem with the laws of physics, is responsible for the order we find in the actual World. This order is of two broad types: chemical and structural. ${ }^{74} \mathrm{We}$ find the structural order in clumpiness, in the fact that matter is densely packed at certain points (e.g. inside stars) and nearly absent at others (e.g. interstellar space). ${ }^{75}$ According to the standard cosmogonical model, this clumpiness originated through the interplay of quantum uncertainty, the tug of gravity, and the expansion of spacetime. ${ }^{76}$ Like all forms of order, the Universe's clumpiness embodies information. Even when all stars burn out and all black holes dissolve, there will still be order (information) in the Universe so long as matter is not uniformly distributed. A state of maximum entropy (zero information-the "heat death") would therefore require eliminating this clumpiness. However, if spacetime expands more rapidly than thermodynamic processes can create disorder, matter will never be uniformly distributed. Therefore, depending on its expansion rate, the Universe could go on storing and processing information forever. Because this logically possible outcome would enhance richness, Leibniz' cosmology predicts it will occur: The Universe will expand so rapidly that order is never lost, but not so rapidly as to destroy itself. ${ }^{77}$

Another point distinguishes Leibnitian cosmology from other theories. If Leibniz is right, then at its beginning the Universe must have been maximally simple. It must have contained as little structure as possible, since structure adds to complexity. On the other hand, to be maximally rich, the Universe in its totality must be replete with diverse, complex, non-repetitive structure. How can a Universe be maximally simple, yet also maximally complex? The only way is for it to change and the general direction of change must be away from simplicity toward complex structure. Since Leibniz' theory requires change, it also requires time. Time is change. We measure space with a ruler; we measure time with a clock. The difference is this: clocks change; rulers do not. Today, time is only an unexplained aspect of nature. Leibniz' theory explains why we experience time.

His theory is also uniquely consistent with the large-scale geometry of our Universe. Our actual Universe began at a moment of maximal simplicity and from that point has expanded continuously, creating structure by expanding. Our past, our future, and the arrow of time that links them all emerge from this universal expansion. ${ }^{78}$ The predicted need of the best possible Universe to originate in maximal simplicity and yet to contain maximal richness explains why we experience these phenomena. Leibniz could not have guessed at the big bang and the subsequent universal expansion, yet his theory implicitly predicts them. His claim that the Universe is at the conjoint maximum of simplicity and richness, is the most parsimonious explanation for the negligible complexity of its actual laws and conditions and the vast richness of its actual phenomena.

\section{Conclusion}

Leibniz' metaphysical conjecture, derived from his notion of God's perfection, generates a coherent, testable description of the best possible Universe. That 
description closely resembles the actual Universe. Further analysis and empirical investigation should explore the possibility that Leibniz' claim might be correct. ${ }^{79}$

\section{Endnotes}

1 Monadology, $\S$ 90, in N. Rescher, G. W. Leibniz's Monadology: An Edition for Students (Pittsburgh: U. of Pittsburgh, 1991), 28.

2 Smolin estimates the chances that randomly selected laws of physics would support long-lived stars as one out of $10^{229}$. L. Smolin, The Life of the Cosmos (New York: Oxford University Press, 1997), 45. Yet long-lived stars are only one of the prerequisites for complex life. See, M. Tegmark, "Is 'the theory of everything' merely the ultimate ensemble theory," Annals of Physics, 270 (1998): 14-21.

3 See, among many other sources: J. Leslie, Universes (London: Routledge, 1989), 25-65; M. J. Rees, Just Six Numbers: The Deep Forces that Shape the Universe (New York: Basic, 2000), 14-163; J. D. Barrow and F. J. Tipler, The Anthropic Cosmological Principle (Oxford: Oxford University Press, 1986, rev. 1988), 219-457; and Smolin, The Life of the Cosmos, $36-46$.

4 Paul Davies coined this usage of "fine-tuning."

5 John Wheeler's "Participatory Anthropic Principle," has intelligent life selecting for wave state reduction a single branch of a Universe that would otherwise remain as an unreduced quantum wave function. See Barrow and Tipler, The Anthropic Cosmological Principle, 505. Smolin suggests that universes emerge through a process akin to Darwinian natural selection. Smolin, The Life of the Cosmos, 36-46.

6 D. Lewis, On the Plurality of Worlds (Oxford: Basil Blackwell, 1986), 2.

7 This is the so-called "weak anthropic principle." For a discussion, see Barrow and Tipler, The Anthropic Cosmological Principle, 15-23.

8 Within the overall multiverse concept, theories differ in nearly every particular. Some have baby universes growing out of black holes; others have multiple universes sprouting from a primordial, pre-cosmic foam; others have our universe expanding and contracting, with the laws of physics changing on each expansion. What these theories and others have in common is an infinitely large, infinite collection of infinitely varied regions, of which our life-supporting region is a very atypical member. For a discussion and critique, see Leslie, Universes, 66-103.

9 W. R. Stoeger, G. F. R. Ellis, and U. Kirchner, "Multiverses and Cosmology: Philosophical Issues," 20 January 2006: http://arxiv.org/PS_cache/astro-ph/pdf/ 0407/0407329.pdf. Arguments for a finite Universe can be found throughout J. Levin, How the Universe Got Its Spots (New York: Random House, 2002).

10 Following Leibniz, this paper will use the word "World" to mean the entire Universe, everything that is. In its lower case form, "world" will sometimes be used to refer to a region of the World.

11 Newton suggested that God might "vary the Laws of Nature, and make worlds of several sorts in several Parts of the Universe." Optics, Part III, Query 31. However, Newton never wrote of the possibility that God might have created the whole Universe on a different plan.

12 G. W. Leibniz, Theodicy, trans. E. M. Huggard (La Salle, Ill.: Open Court, 1985) § 7, 127.

13 Most theologians would accept this description. See T. V. Morris, ed., The Concept of God (Oxford: Oxford University, 1987), 6-8.

14 Monadology, § 55, in Rescher, G. W. Leibniz's Monadology, 24. Philosophers refer to Leibniz' belief that God would do nothing without a reason as his "principle of sufficient reason."

15 A. Flew, An Introduction to Western Philosophy: Ideas and Argument from Plato to Popper (New York: Thames \& Hudson, 1989), 240. 
16 "Discourse on Metaphysics," § 6, in G. W. Leibniz, Philosophical Texts ("Texts"), trans. R. S. Woolhouse and R. Francks (Oxford: Oxford University Press, 1998), 58.

17 "Principles of Nature and of Grace, Founded on Reason," § 10, in G. W. Leibniz, Philosophical Writings ("Writings"), trans. M. Morris and G. H. R. Parkinson (London: Orion, 1973), 200.

18 "A Resume of Metaphysics," in Leibniz, Philosophical Writings, 144.

19 Two errors made by many of Leibniz' critics, including Voltaire, are to focus on whether the World is best for us (humans) and to focus on conditions on planet Earth. Leibniz rejected both of these concerns as anthropocentric.

20 N. Rescher, On Leibniz (Pittsburgh: University of Pittsburgh, 2003), 27.

21 Leibniz, Theodicy, § 208, 257.

22 Rescher, On Leibniz, 28.

23 Quoted in J. Horgan, Rational Mysticism: Spirituality Meets Science in the Search for Enlightenment (New York: Houghton Mifflin, 2000), 172.

24 Carl Sagan and Ann Druyan, "On Earth as it is in heaven-origin of the Earth and the solar system," Omni, Oct. 1992.

25 M. Livio, The Accelerating Universe (New York: John Wiley, 2000), 260-265. Livio defines the Copernican principle to mean that a valid theory cannot "require humans to occupy a very special place or time for it to work." Ibid., 30. All multiverse theories contradict this principle.

26 M. Gell-Mann, The Quark and the Jaguar (New York: Freeman, 1994), 99-100.

27 A. Zee, Fearful Symmetry (Princeton: Princeton University, 1999), 211.

28 P. Davies, "Teleology Without Teleology," in In Whom We Live and Move and Have Our Being: Panentheistic Reflections on God's Presence in a Scientific World, eds. P. Clayton and A. Peacocke (Grand Rapids, Mich.: William B. Eerdmans, 2004), 106. In this same article, Davies suggests that our laws of physics "may form an optimal set" in the degree to which they optimize organizational complexity. Ibid., 104. As an example, he suggests that the way quantum mechanics assigns probabilities to components of the wave function may "optimize some information theoretic quantity, such as the mutual information between components of entangled physical systems." Ibid., 302, n. 26.

29 The points made in this paragraph are from Rescher, On Leibniz, 57-59.

30 Leibniz assumed that every event is determined in its detailed entirety by the laws of physics and initial conditions of the Universe. If this were true, the Universe and its laws and initial conditions would be in some sense the same. The former would simply be the rolling out of the latter. This paper does not assume this true. It focuses on whether Leibniz' richest/simplest hypothesis is a reasonable explanation of our physical laws and initial conditions, whether the Universe those laws and conditions generate is deterministic or not.

31 For a more detailed description, see Gell-Mann, The Quark and the Jaguar, 34-41.

32 Another limitation is that, because of inherent limitations on the process of mathematical reasoning, AIC can only give a maximum value for the complexity of the measured system. A system might be less complex than we think it is because we might not have thought of a simpler way to describe it.

33 See Gregory Chaitin, "On the intelligibility of the universe and the notions of simplicity, complexity and irreducibility," presented at a meeting of the German Philosophical Society, Bonn, September 2002.

34 Werner Heisenberg, quoted in Livio, The Accelerating Universe, 35.

35 Quoted in Rescher, On Leibniz, 33.

$36 \mathrm{~J}$. Barbour and L. Smolin, "Extremal variety as the foundation of a cosmological quantum theory," published on the web at http://arxiv.org/abs/hep-th/9203041. The authors intended the quantity they presented in this paper (variety) to reflect Leibnitian richness, at least in a static system. Barbour explains this further in J. Barbour, "The Deep and Suggestive Principles of Leibnizian Philosophy," The Harvard Review of Philosophy, XI (2003): 45-58. 
37 Barbour and Smolin, "Extremal variety as the foundation of a cosmological quantum theory," 3.

38 Each additional dot, house, or particle adds one over some number to total variety. On the other hand, each may also increase the absolute indifference of some existing particle. Thus, variety cannot be calculated for an infinitely large universe. In such a World, an infinite amount of information would be needed to specify any particular neighborhood. Both the numerator and the denominator of the fractional part of the equation would be indeterminately large, yielding a meaningless result.

39 Barbour and Smolin, "Extremal variety as the foundation of a cosmological quantum theory," 1.

40 I owe this example to an anonymous reviewer.

41 Imagine a woodlot that hosts twelve unique species. A farmer cuts down half of it and plants a field that attracts two non-woodland species. The alpha diversity of the woodlot is higher than that of the field, but the beta diversity of field and woodlot together is higher than that of the woodlot alone. Gamma diversity tries to capture the diversity of larger regions. See, R. H. Whittaker, "Evolution and measurement of species diversity," Taxon, 21 (1972): 213-251.

42 Barbour and Smolin, "Extremal variety as the foundation of a cosmological quantum theory," note (at 22) that, "the actual definition we used for the variety does not change the qualitative results, although the detailed form of the extremal solutions are, of course, different."

43 Barbour and Smolin note that the dots in their highest-variety graphs tend to cluster in tightly packed groups, leaving vast empty spaces between them. This is an example of a higher-order, static characteristic.

44 I owe this suggestion to a personal communication from Julian Barbour.

45 Barbour and Smolin, "Extremal variety as the foundation of a cosmological quantum theory," 28-29. Barbour and Smolin ran computer simulations with dots that move randomly. The program stops these movements whenever the system attains a higher variety, and begins again with that point as the marker. In this stepwise fashion, the system moves stochastically toward maximal variety. In extremal variety configurations, both a long-ranged, attractive force that causes dots to cluster in groups and a short-ranged, repulsive force that prevents two dots from occupying the same spot seem to emerge. The authors liken the long-range force to gravity and the short-range force to the Pauli exclusion principle.

46 For example, atomic structure. Barbour now believes that the project of reducing the laws of physics to a continuous, global tendency toward higher variety is unlikely to succeed. Barbour, "The Deep and Suggestive Principles of Leibnizian Philosophy."

47 C. Darwin, The Origin of Species, 6th ed. (Chicago: University of Chicago, 1952), 243.

48 Leibniz, Theodicy, § 8, 128.

49 Rescher, On Leibniz, 27 (emphasis in original). The quote is Rescher's explanation of Leibniz' claim.

50 Variety is a pure number (like dimensionality or the gravitational constant). AIC can be given in bits. It makes intuitive sense to speak of one possible universe as having higher variety per bit of its foundational hypotheses (its laws plus its initial conditions) than another. Alternatively, we could define the variety of the actual Universe as equalling one Leibniz and designate that as our unit of variety. Then we could state the World merit of our actual Universe and other possibilities in terms of fractional Leibnizes per bit of "hypotheses."

51 See note 7, supra.

52 Not everyone agrees. See the extended discussion in Leslie, Universes, 104-149.

53 To take just one example, it does not seem that a weakening or strengthening of the gravitational constant by one tenth of one per cent would preclude our existence. 
54 Rees suggests a similar approach, using a different criterion of specialness. See M. Rees, Our Cosmic Habitat (Princeton: Princeton University, 2001), 175-177. SEK (at 23) criticize Rees' criterion.

55 S. Wolfram, A New Kind of Science (Champaign, Ill.: Wolfram Media, 2002), 470.

56 E. J. Squires, "Do we live in the simplest possible interesting world?" European Journal of Physics, vol. 2(1981): 55-57.

57 Quoted in Livio, The Accelerating Universe, 34. More specifically, the principle of relativity emerged from Einstein's stubborn insistence (following Leibniz' principle of sufficient reason) that the laws of physics would not differ in different frames of reference unless there were a reason for them to do so.

58 P. Davies, Superforce (New York: Simon \& Schuster, 1984), 8.

59 L. Pearce Williams, “Ether," Encyclopedia Americana, vol. 10 (1994): 608-609.

60 H. Pagels, Perfect Symmetry (London: Joseph, 1985), 347.

61 Tegmark, "Is 'the theory of everything' merely the ultimate ensemble theory," 8.

62 Ibid., 2.

63 Quoted in A. O. Lovejoy, The Great Chain of Being (Cambridge, Mass.: Harvard University, 1964, orig. ed. 1936), 179.

64 Tegmark, "Is 'the theory of everything' merely the ultimate ensemble theory," 4.

65 Ibid.

66 On the same point, see Rees, Our Cosmic Habitat, 175.

67 S. Hawking, A Brief History of Time: Tenth Anniversary Edition (New York: Bantam, 1996), 130.

68 See Levin, How the Universe Got Its Spots, 178-195, for a detailed explanation of the possibility described in this paragraph.

69 N. J. Cornish et al., "Constraining the Topology of the Universe," Physical Review Letters, vol. 92, no. 20 (21 May 2004): 201302.

70 See generally, Livio, The Accelerating Universe.

71 Of course, there is much that we do not know about the future of the Universe. The largely unknown forces behind the accelerating expansion may someday reverse course. Or the acceleration could prove to be too great, resulting in a "big rip" that would pull apart even the molecules in our bodies. See R. R. Caldwell, M. Kamionkowski, and N. N. Weinberg, "Phantom Energy and Cosmic Doomsday," http://www.arxiv.org/ PS_cache/astro-ph/pdf/0302/0302506.pdf. However, if Leibniz is correct, neither of these scenarios will occur.

72 An anonymous reviewer raised this point.

73 The points in this paragraph and the next two are largely drawn from D. Layzer, Cosmogenesis: The Growth of Order in the Universe (New York: Oxford, 1990), 27-28, 138-145.

74 Chemical order derives in large part from the fact that most baryonic matter consists of hydrogen. This happened because of the speed of the universal expansion. See Layzer, Cosmogenesis, 141-146.

75 See note 43 , supra.

76 B. Greene, The Fabric of The Cosmos: Space, Time, and the Texture of Reality (New York: Random House, 2004), 304-308.

77 This also implies that dark energy is not what Caldwell, Kamionkowski and Weinberg call "phantom energy." See note 71, supra.

78 See Greene, The Fabric of The Cosmos, 143-176.

79 The author wishes to thank Nicholas Rescher and Julian Barbour for valuable comments.

\section{Biographical Notes}

William Lane is a graduate of Yale (B.A.) and Columbia (J.D.). He is a lawyer who resides in Woodstock, Virginia. He can be reached at lane2125@yahoo.com. 
